\title{
Photoacoustic tomography: Ultrasonically beating optical diffusion and diffraction (Presentation Video)
}

Lihong Wang

Lihong Wang, "Photoacoustic tomography: Ultrasonically beating optical diffusion and diffraction (Presentation Video)," Proc. SPIE 8943, Photons Plus Ultrasound: Imaging and Sensing 2014, 894334 (18 March 2014); doi: $10.1117 / 12.2064189$

SPIE. Event: SPIE BiOS, 2014, San Francisco, California, United States 


\title{
Photoacoustic tomography: Ultrasonically beating optical diffusion and diffraction (Presentation Video)
}

\author{
Lihong Wang, Washington Univ. in St. Louis (United States)
}

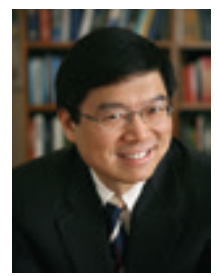

\section{BIOS 2014 Hot Topics Presentation}

A decade of research has pushed photoacoustic computed tomography to the forefront of molecular-level imaging, notes SPIE Fellow Lihong Wang (Washington University, St. Louis) in his plenary talk, "Photoacoustic Tomography: Ultrasonically Beating Optical Diffusion and Diffraction."

Modern optical microscopy has resolution and diffraction limitations. But noninvasive functional photoacoustic computed tomography has overcome this limit, offering deep penetration with optical contrast and ultrasonic resolution of $1 \mathrm{~cm}$ depth or more -- up to $7 \mathrm{~cm}$ of penetration in some cases, such as evaluating sentinel lymph nodes for breast cancer staging. This opens up applications in whole body imaging, brain function, oxygen saturation, label-free cell analysis, and noninvasive cancer biopsies.

View presentation video on SPIE's Digital Library: http://dx.doi.org/10.1117/12.2064189 